\title{
Adaptive changes in the gut microbiome during standard-of-care chemoradiotherapy for gynecologic cancers
}

Molly B. EL ALAM, MPH ${ }^{1 *}$; Travis T. SIMS, MD, MPH ${ }^{2 *}$; Ramez KOUZY, MD ${ }^{1}$; Greyson W. G. BIEGERT, BS $^{1}$; Joseph ABI JAOUDE, MD ${ }^{1}$; Tatiana V. KARPINETS, PhD ${ }^{3}$; Kyoko YOSHIDA-COURT, PhD ${ }^{1}$; Xiaogang WU, PhD ${ }^{3}$; Andrea Y. DELGADO MEDRANO, BS ${ }^{1}$; Melissa P. MEZZARI, $\mathrm{PhD}^{4}$; Nadim J. AJAMI, PhD'3; Travis SOLLEY, BS ${ }^{1}$; Mustapha AHMED-KADDAR, BS ${ }^{1}$; Lilie L. LIN, MD ${ }^{1}$; Lois RAMONDETTA, MD²; Amir JAZAERI, MD²; Anuja JHINGRAN, MD' ${ }^{1}$; Patricia J. EIFEL, MD ${ }^{1}$; Kathleen M. SCHMELER, MD²; Jennifer WARGO, MD, MMSc ${ }^{5}$; Ann H. KLOPP, MD, PhD ${ }^{1+}$; Lauren E. COLBERT, $M D, M^{1+}$

\footnotetext{
${ }^{1}$ Department of Radiation Oncology, The University of Texas MD Anderson Cancer Center, Houston, TX, USA. ${ }^{2}$ Department of Gynecologic Oncology and Reproductive Medicine, The University of Texas MD Anderson Cancer Center, Houston, TX, USA. ${ }^{3}$ Department of Genomic Medicine, The University of Texas MD Anderson Cancer Center, Houston, TX, USA. ${ }^{4}$ Department of Molecular Virology and Microbiology, Alkek Center for Metagenomics and Microbiome Research, Baylor College of Medicine, Houston, TX, USA. ${ }^{5}$ Department of Surgical Oncology, The University of Texas MD Anderson Cancer Center, Houston, TX, USA.

*These authors contributed equally

+Shared corresponding Authorship
}

Correspondence: L.E. Colbert or A.H. Klopp, Department of Radiation Oncology, Unit 1422, The University of Texas MD Anderson Cancer Center, 1515 Holcombe Boulevard, Houston, TX 77030, USA. Telephone: 832-652-6033 (L.E.C.), 713-563-2444 (A.H.K); fax: 713-745-2398; e-mail: Icolbert@mdanderson.org, aklopp@mdanderson.org.

Conflicts of Interest: The authors report no conflicts of interest.

Financial Support: This research was supported in part by the Radiological Society of North America Resident/Fellow Award (to L.E.C.); by the National Institutes of Health (NIH) through MD Anderson's Cancer Center Support Grant P30CA016672 and through T32 grant CA101642-14 (T.T.S.); and by The University of Texas MD Anderson Cancer Center HPV-related Cancers Moonshot (L.E.C., A.K.). The funding sources were not involved in the development of the research hypothesis, study design, data analysis, or manuscript writing. Data access was limited to the authors of this manuscript. 
1

2 Condensation: The gut microbiome changes significantly during pelvic chemoradiation, 3 which should be considered in future studies.

4 Short Title: Gut microbiome changes during pelvic chemoradiation

\section{AJOG at a Glance:}

6 A. Why was this study conducted? To determine changes in gut microbiome associated

7 with treatment of gynecologic cancers

8 B. What are the key findings? The gut microbiome changes significantly during

9 treatment, and variably returns to baseline. Approximately $2 / 3$ of patients return to or

10 near their baseline gut diversity, while $1 / 3$ remain significantly less diverse. The

11 composition of nearly all patients' guts is significantly different after CRT.

12 C. What does this study add to what is already known? This study reports the largest

13 cohort of serial sequencing before, during and after CRT to inform future studies. 


\section{ABSTRACT}

18 Background: A diverse and abundant gut microbiome can improve cancer patients'

19 treatment response; however, the effect of pelvic chemoradiotherapy (CRT) on gut

20 diversity and composition is unclear. The purpose of this prospective study was to

21 identify changes in the diversity and composition of the gut microbiome during and after

22 pelvic CRT.

23 Materials and Methods: Rectal swabs from 58 women with cervical, vaginal, or vulvar

24 cancer from two institutions were prospectively analyzed before CRT (baseline), during

25 CRT (weeks 1, 3, and 5), and at first follow-up (week 12) using 16Sv4 rRNA gene

26 sequencing of the V4 hypervariable region of the bacterial $16 \mathrm{~S}$ rRNA marker gene.

27 Observed operational taxonomic units (OTUs; representative of richness) and Shannon,

28 Simpson, Inverse Simpson, and Fisher diversity indices were used to characterize alpha

29 (within-sample) diversity. Changes over time were assessed using a paired t-test,

30 repeated measures ANOVA, and linear mixed modeling. Compositional changes in

31 specific bacteria over time were evaluated using linear discriminant analysis effect size.

32 Results: Gut microbiome richness and diversity levels continually decreased throughout

33 CRT (mean Shannon diversity index, 2.52 vs. 2.91; all $P<0.01$ ), but were at or near

34 baseline levels in $60 \%$ of patients by week 12 . Patients with higher gut diversity at

35 baseline had the steepest decline in gut microbiome diversity. Gut microbiome

36 composition was significantly altered during CRT, with increases in Proteobacteria and

37 decreases in Clostridiales, but adapted after CRT, with increases in Bacteroides

38 species.

39 Conclusion: After CRT, the gut microbiome's diversity tends to return to baseline levels,

40 but its structure and composition remain significantly altered. These changes should be 
41 considered when designing studies to analyze the gut microbiome as a predictive or

42 prognostic biomarker in patients who receive pelvic CRT for gynecologic cancers.

43

44 Key words: gynecologic cancer, microbiome, chemoradiation 


\section{INTRODUCTION}

47 The current standard of care for patients with human papillomavirus (HPV)-related

48 gynecologic cancers is concurrent chemoradiotherapy (CRT), which is generally

49 followed by brachytherapy ${ }^{3}$. Despite such therapy, approximately $30-40 \%$ of patients

50 develop recurrent disease.

Potentially relevant biomarkers for predicting treatment response in gynecologic

52 cancer patients include tumor genomic markers, immune response markers, and tumor

53 microenvironment markers. One intriguing tumor microenvironment marker is the

54 microbiome, which may affect immune response during and after CRT. The microbiome

55 is composed of various communities of bacteria, fungi, viruses, and archaea that coexist

56 with their host $\stackrel{4,5}{ }$. Gut microbiota have been shown to affect host immunity, play a role in

57 carcinogenesis, and influence therapy response $\frac{6-9}{}$. At least one study has shown that

58 more diverse and abundant gut microbiota can improve immunotherapy response $\underline{10}$.

59 Because HPV-related cancers are primarily mucosal and virally induced, the

60 microbiome may play a significant role in these tumors' response to treatment. The gut

61 is the largest immune organ in the body, and mucosa play a key role in regulating

62 immune response to outside pathogens in conjunction. Determining the gut

63 microbiome's role in influencing treatment response in gynecologic cancer patients

64 requires an improved understanding of the ways in which CRT itself affects the gut

65 microbiome.

The gut microbiota of cervical cancer patients and healthy individuals differ

67 significantly, ${ }^{12}$ and increasing data suggest that the gut microbiome plays a role in

68 gynecologic cancer including, but not limited to, treatment response and survival11. Like

69 patients with gynecologic cancers, patients with other cancers, including anal, rectal, 
prostate, cervical, and endometrial cancer, are treated primarily with pelvic

71 radiotherapy, with or without chemotherapy, and likely have similar temporal changes in

72 the diversity and composition of the gut microbiome over time as a result of CRT;

73 However, prospective data on the changes the gut microbiome undergoes during pelvic

74 radiotherapy remain scarce, as most studies on the subject have provided only single

75 time-point or retrospective data. Having prospective data is particularly important for

76 studying the temporal changes in the gut microbiome. We hypothesize that CRT could

77 induce structural and possibly compositional changes in the gut microbiome of patients

78 with gynecologic cancers. The purpose of this study was to prospectively determine the

79 effect of standard pelvic CRT on the gut microbiome in a cohort of women with locally

80 advanced gynecologic cancers. Our findings could be used to guide future studies of

81 the gut microbiome as a predictive and prognostic biomarker.

MATERIALS AND METHODS

$\underline{\text { Patients }}$

Under an Institutional Review Board-approved protocol (MDACC 2014-0543), we

prospectively collected rectal swab specimens from 58 gynecologic cancer patients who

87 received standard-of-care pelvic CRT at MD Anderson or the Lyndon B. Johnson

88 Hospital Oncology Clinic between 2015 and 2019. Consented patients with newly

89 diagnosed, biopsy-proven locally advanced cervical, vaginal, or vulvar carcinoma were

90 eligible for the study. Patients who previously received pelvic radiation or systemic

91 therapy were excluded. Eligible patients received CRT with external beam radiotherapy

92 and weekly cisplatin, which was followed by brachytherapy if indicated. All patients

93 received a minimum radiation dose of 45 Gy given in 25 fractions over 5 weeks along 
94 with cisplatin at $40 \mathrm{mg} / \mathrm{m}^{2}$ and either 2 pulsed dose rate brachytherapy treatments or 5

95 high dose rate brachytherapy treatments. The majority of patients were tested for the

96 Human Papillomavirus (HPV). Patients' antibiotic use during the study period was

97 determined by searching inpatient and outpatient pharmacy prescription

98 records. Demographic data was also collected from patients' medical records.

\section{Sample collection, processing, and sequencing}

101 Rectal swab samples were collected immediately before treatment initiation and 1, 3, 5, 102 and 12 weeks after treatment initiation. Week 1 samples were collected only after 103 patients received 1-5 fractions of radiotherapy. We collected samples using quickrelease matrix designed Isohelix DNA swabs. Lysis buffer (400 mL) was added to each

105 sample within 1 hour of collection, and all samples were stored at $-80^{\circ} \mathrm{C}$ until they were 106 subjected to RNA extraction and sequencing $\underline{12}$.

107 Sequencing of the V4 hypervariable region of the bacterial 16S rRNA marker 108 gene (16Sv4) was performed. The $16 \mathrm{~Sv} 4$ gene is both the most conserved and the 109 most variable segment of the bacterial genome, making it the ideal target for performing 110 phylogenetic analysis. 16S performed at the Alkek Center for Metagenomics and

111 Microbiome Research at Baylor College of Medicine using a methodology from the

112 Human Microbiome Project $\underline{13}$. The sequencing was performed as described previously

113 using strict OTU assignment criteria for rigorous classification $\underline{12}$. Operational taxonomic

114 unit (OTU) assignment was performed using clustering with $97 \%$ sequence similarity

115 using in-house pipelines developed for the human microbiome project. 
118 We evaluated all available alpha (within-sample) diversity metrics, including richness

119 (the absolute number of observed OTUs), Shannon diversity, Inverse Simpson diversity,

120 Simpson diversity, and Fisher diversity. We used the paired Student t-test to compare

121 the mean diversity metrics recorded throughout treatment to the mean baseline diversity

122 metrics. To compare changes in gut richness and diversity across the first 4 time points

123 for patients who provided samples at those times $(n=23)$, we performed repeated

124 measures analysis of variance (ANOVA) with Bonferroni correction for post hoc

125 comparisons to identify timepoints that had different alpha diversities. For each patient

126 who provided samples at all five time points $(n=17)$, we tracked the change in diversity

127 from baseline to week 5 to week 12 to determine the number of patients for whom gut

128 diversity returned to baseline. For patients who did not provide samples at all time

129 points $(n=58)$, we built a linear mixed model for time as a predictor of diversity.

130 Covariates were time, and an interaction term of baseline diversity and time, with

131 individual patients as a random effect. To determine whether taking antibiotics affected

132 the diversity of the gut microbiome, we collected information on patients' use of

133 antibiotics prior to weeks 1, 3, and 5 and performed an independent Student t-test to

134 compare the means of each diversity metric at each time point between patients who

135 took antibiotics before that time point and patients who did not. We also performed an

136 independent Student t-test to determine whether the fold change in diversity from

137 baseline to week 12 was dependent on prior antibiotic use.

139 Assessment of gut composition over time

140 We used linear discriminant analysis effect size $e^{14}$ to examine beta (between-sample)

141 diversity and identify changes in bacterial genera between baseline and week 5 . To 

visualize the differences in gut microbiota between baseline and week 5 , we calculated an OTU enrichment index, which is specifically designed to evaluate enrichment in the

144 presence of rare species, as described previously $\underline{6}$. The enrichment index can take

145 values ranging from -1 (OTU found in week 5 but not baseline) to 1 (OTU found in

146 baseline but not week 5). We generated a heatmap of OTU abundances, in which the

147 columns represented samples and the rows represented the OTUs, and used the

148 overall abundance distribution of all OTUs to identify thresholds for low (green), medium

149 (yellow), and red (high) abundances. Statistical significance was set at an alpha of $5 \%$

150 for a two-sided P-value. Analyses were conducted using RStudio 1.2.5033 15 .

\section{RESULTS}

\section{Patients}

154 Patient characteristics are provided in supplementary table 1. Our patient population

155 included 58 patients $(55,2$, and 1 with cervical, vulvar, and vaginal cancer,

156 respectively). The patients' mean age was 49.36 years (standard deviation [SD], 10.52

157 years). Of the 58 patients, 42 took antibiotics during the study period, of whom 8

158 patients received antibiotics prior to week 1, 13 patients prior to week 3 , and 18 patients

159 prior to week 5 (Supplemental Figure 1). Twelve patients received only

160 sulfamethoxazole trimethoprim with brachytherapy at week 5.

Most patients $(79.3 \%)$ had squamous cell carcinoma. Of the 56 patients whose

162 HPV status we determined, 82\% were positive for the virus. Baseline samples were

163 collected from 55 patients; week 1 samples, from 39 patients; week 3 samples, from 37

164 patients; week 5 samples, from 47 patients; and week 12 samples, only 16 patients

165 (Supplemental Table 2). 
167 Gut richness and diversity over time

168 Overall, the gut richness and diversity metrics significantly decreased by week 5 but 169 returned to baseline levels after CRT. The baseline means for observed OTUs and the 170 Shannon, Simpson, Inverse Simpson, and Fisher diversity indices were 107.58 (SD, $17134.60), 2.91$ (SD, 0.59), 0.87 (SD, 0.09), 11.39 (SD, 6.55), and 18.26 (SD, 6.98),

172 respectively (Table 1). None of the alpha diversity metrics at week 1 or week 3 differed 173 significantly from those at baseline (Table 1; $P>0.05$ for all); However, the alpha 174 diversity metrics of the week 5 samples were significantly lower than those of the 175 baseline samples: the mean observed OTU value was $83.79(P<0.001)$; the mean 176 Shannon diversity index, $2.52(P<0.001)$; the mean Simpson diversity index, $0.81(P=$ 177 0.002); the mean Inverse Simpson diversity index, $8.23(P=0.001)$; and the mean 178 Fisher diversity index, $13.53(P<0.001)$. The repeated measures ANOVA test showed, 179 that the mean observed OTUs at week 5 (81.91) were significantly lower than those at 180 baseline (108.39; $P=0.007$ ), week 1 (mean, 103.91; $P=0.048)$, and week 3 (mean, $18197.17 ; P=0.024)$ (Figure 1A). Furthermore, Fisher diversity index was lower at week 5 182 than at baseline $(P=0.008)$, week $1(P=0.048)$, and week $3(P=0.026)$ (Figure 1C). 183 Although the Shannon, Simpson, and Inverse Simpson diversity indices decreased 184 between baseline and week 5 , they did not differ significantly between week 5 and 185 either week 1 or 3 (Figure 1B, D, E). We found no significant differences in diversity 186 metrics between patients who took antibiotics prior to each time point and those who 187 didn't.

The alpha diversity metrics at week 12 were not statistically lower than those at 189 baseline $(P>0.05$ for all). Of the 11 patients who provided samples at baseline, week 5 , 

and week 12 (Figure 2), 4 (36\%) had higher diversity at week 12 than at baseline, 4

191 (36\%) had increased diversity after CRT that did not return to baseline levels, and 3 $192(27 \%)$ had a steady decrease in their microbiome diversity over time until follow up.

193 Overall, two-thirds of patients' diversity indices returned to near-baseline levels. We

194 found no association between antibiotic use and gut microbiome diversity.

(Table 2) both confirmed the above findings $(P<0.01)$ and showed that diversity decreased significantly with time. In addition, for nearly all diversity indices, there was an interaction effect between baseline diversity and time $(P<0.01)$, indicating that

199 baseline diversity is important in predicting the response of the gut microbiome to CRT

200 (Figure 3). Patients with high baseline diversity metrics had steeper declines in their gut 201 diversity over time than those with low baseline diversity metrics did.

202

Gut composition over time

Using linear discriminant analysis effect size, we identified compositional

changes in patients' gut microbiomes based on specific bacterial phyla, classes, orders,

206 families, and genera that were differentially enriched in patients between baseline and

207 week 5 (Figure 4A,B), between week 5 and week 12 (Figure 4C), and between

208 baseline and week 12 (Figure 4D) (false discovery rate $P<0.05$; linear discriminant 209 analysis score $>4$ ). During CRT, the relative abundances of Clostridia, Clostridiales,

210 Faecalibacterium, and Ezakiella decreased, whereas the relative abundances of

211 Proteobacteria, Gammaproteobacteria, Bacilli, Pasteurellales, Pasteurellaceae, and

212 Haemophilus increased (Supplementary Figure 2). Between week 5 and week 12, the

213 abundances of Proteobacteria and Gammaproteobacteria continued to decrease, 
whereas that of Ezakiella returned to its baseline level. Still, we saw significant and week 12 (Supplementary Figure 3).

218 increase in mostly pathogenic $\frac{16}{}$, gram-negative Proteobacteria (Figure 5A) and a

219 decrease in generally beneficial Clostridia (Figure 5B), both of which tended to return to

220 baseline levels after CRT. Thus, the most significant long-term alteration was an

221 decrease in Bacteroidetes (Figure 5C), which likely helped prevent pathogenic bacteria

222 from colonizing the gut and was a beneficial adaptation in response to a significant

223 assault on the gut microbiome.

Rare species enrichment analysis (Figure 5D-F) demonstrated an adaptation of

225 rare species at the OTU level. The relative abundance of Clostridia as a class

226 significantly decreased from baseline to week 5, whereas the pattern of changes of rare

227 individual OTUs were more complex. Most levels of rare Clostridia species were

228 significantly higher at baseline than at week 5. However, a small fraction of individual

229 OTUs of Clostridia, increased their occupancy during CRT (Figure 5E).

\section{DISCUSSION}

\section{Principal Findings}

233 We hypothesized that the process of undergoing standard-of-care CRT induces

234 compositional and/or functional changes in the gut microbiome that are relevant for

235 analysis of the gut microbiome as a future biomarker. Our prospective longitudinal

236 analysis showed that gut microbial diversity is stable immediately after the initiation of

237 CRT but declines significantly over the first 5 weeks of treatment. After patients 
complete CRT, their gut diversity returns to baseline levels, but their gut composition

and structure are altered.

Similarly, our findings that alterations in microbial composition and relative

241 abundance occur within the first 5 weeks of CRT initiation and after CRT completion

242 corroborate those of animal studies ${ }^{21}$. Furthermore, our study builds upon data reported

by Nam et al., ${ }^{19}$ who established that pelvic radiotherapy alters the composition of the

244 gut microbiome of gynecologic cancer patients.

Our findings also provide additional insight into the temporal changes of the

247 that we observed is a unique effect that may influence the clinical outcomes of

249 beyond the scope of the current study, but this mechanism could be related to changes

250 in the gut epithelium and mucus layer that are followed by the overgrowth of pathogenic

251 bacteria, which could in turn affect immune cells' maturation and responses to tumor.

252 Cumulative fractions of CRT may induce the outgrowth of these radioresistant or

253 pathologic microbial taxa, resulting in the selection of taxa that are tolerant to radiation-

254 induced insults.

Our findings show that baseline gut diversity is a strong predictor of the degree of

256 change in gut diversity during CRT. Compared with patients with low gut diversity at

257 baseline, those with high gut diversity at baseline had a greater decline in gut diversity

258 from baseline to week 5. This was an unexpected finding because gut diversity is

259 commonly seen as a marker of vulnerability and immunogenicity,,$\frac{6,20}{2}$ and high gut

260 diversity is thought to be beneficial. We expected to see an overall decline in gut

261 diversity at 5 weeks in all patients, regardless of their baseline gut diversity, but this 
262 finding suggests that the best target group for intervention may actually be patients with

263 high baseline gut richness and diversity, rather than those with low baseline richness

264 and diversity. We did not specifically investigate these high-diversity patients' gut

265 composition in the present study, and larger studies are warranted to investigate the

266 impact of gut composition on maintaining diversity.

CRT significantly impacted gut microbial composition in this longitudinal analysis.

Strong adaptations of microbiota composition after CRT were observed in almost every

patient. The gut microbiome is intrinsically dynamic $\stackrel{21}{ }$, and this should be taken into

account when considering how CRT impacts its composition over time. Here we show

271 that the gut microbiome shifts from its baseline state towards a new composition that is

272 represented by the relative abundance of different taxa. Specifically, we found that a

273 variety of bacterial species, namely Clostridiales and Proteobacter, were significantly

274 altered during CRT but returned to baseline levels following the completion of the

275 therapy. Other species, namely Bacteroidetes, remained permanently altered.

276 Interestingly, preliminary investigations have shown that the relative abundance of

277 Clostridia may serve as a potential marker for human health and cancer treatment

278 response 22,23 .

279 Our longitudinal data demonstrate the variability of the gut microbiome during

280 CRT; as both pathogenic and beneficial organisms respond to the shifting

281 microenvironment, the abundance of certain taxa expands while that of others declines.

282 Importantly, the bacterial community 3 months after treatment remained altered as

283 compared with that at baseline, indicating that the gut microbiome of the patients who

284 completed CRT did not fully recover over this time, despite possible oncologic clinical

285 recovery. This appears to be due to a prevalence of pathogenic bacteria and a 
286 decrease of beneficial bacteria during CRT, followed by an adaptive shift in

287 Bacteroidetes to overgrow these pathogenic bacteria and allow beneficial Clostridiales

288 to return to baseline levels. Others have reported similar findings in patients undergoing

289 fecal microbiota transplantation 21,24 . In our analysis, we accounted for the effect of

290 antibiotic use on the gut microbial profiles, but we were unable to account for the effects

291 of patients' chemotherapy, other medications, or diet, which should be considered in

292 future studies. However, our findings support the notion that in some patients, certain

293 therapeutic interventions can lead to the permanent divergence of the gut microbiome.

295 Clinical Implications

Our findings corroborate limited existing research showing that CRT diminishes

297 the gut microbiome in cancer patients. Up to $20 \%$ of patients who have had CRT have 298 long-term diarrhea after $\mathrm{CRT}^{25}$, and it is possible these changes are related to lack of 299 re-establishment of gut microbiome. Future studies should examine long-term gut 300 toxicity for these patients. It is also important to note that the administration of antibiotics 301 for treatment-related toxicities did not significantly alter the gut microbiome in this study 302 and should be safe during pelvic CRT.

The results of our study not only show that intentionally modifying the gut

304 microbiota to accumulate CRT-tolerant species could be used as an interventional 305 strategy to reduce treatment toxicity but also show that such modification should be 306 tailored to individual patients. Researchers in diverse areas of medicine have studied 307 the treatment-enhancing and toxicity-limiting utility of the gut microbiome ${ }^{20,21}$. In one 308 murine model, radiation-induced dysbiosis increased susceptibility to radiotherapy309 related gastrointestinal toxic effects ${ }^{26}$. One such effect, chronic radiation enteritis, rarely 
occurs after pelvic CRT but can severely affect quality of life. Although epithelial

311 disruption and damage certainly play a role in radiation enteritis, a paucity of "good

312 bacteria," such as Clostridiales, might also potentiate chronic enteritis. One recent study

313 suggested that fecal microbiota transplantation might improve severe radiation enteritis,

314 which raises the possibility that replacing bacterial populations with more normally

315 balanced populations could be curative ${ }^{27}$. Wang et al. $\underline{26}$ recently reported the first case

316 series of patients with immune checkpoint inhibitor-associated colitis successfully

317 treated with fecal microbiota transplantation. We have also previously witnessed the

318 effect of low microbiome diversity on increased acute radiation toxicity ${ }^{13}$. Regardless of

319 these findings, determining whether changes in the human gut microbiome during CRT

320 affect patients' risk of treatment-related toxic effects warrants further investigation.

322 Strengths and Limitations

323 The present study had some limitations. First, because this study included

324 patients at two institutions within the greater Houston area, its results might not be

325 generalizable to a broader geographic population. Still, our study is one of the largest

326 longitudinal analyses of changes in the gut microbiome in patients with gynecologic

327 malignancies. Second, owing to the longitudinal study design, some of the patients were

328 inevitably lost to follow-up. To account for this loss, we performed rigorous statistical

329 testing; and regardless, longitudinal sampling remains one of the most robust

330 approaches to analyzing microbiome data. We also followed a set protocol from sample

331 collection through $16 \mathrm{~S}$ sequencing and controlled for batch effects with each

332 sequencing run in order to limit artifactual variations. 
334 treatment response and prognosis and guide future studies of interventions for chronic

335 radiation enteritis in the case of gynecologic malignancies. The serial changes in the gut

336 microbiome during CRT we present should be considered when designing studies

337 aimed at analyzing the effects of the gut microbiome on treatment response and 338 toxicity.

340 Acknowledgments

341 We thank the patients who participated in the study. We would like to acknowledge Mr.

342 Joe Munch on his editorial support on this manuscript.

\section{Author contributions}

345 All authors were involved in patient identification; data collection, analysis, and

346 interpretation; and review and approval of the final manuscript. The study was

347 conceived by L.E.C., A.K., T.T.S., and M.B.E.A. The manuscript was written by T.T.S

348 and M.B.E.A. 


\section{References}

353 1. Arbyn M, Weiderpass E, Bruni L, et al. Estimates of incidence and mortality of

354 cervical cancer in 2018: a worldwide analysis. The Lancet Global Health.

355 2020;8(2):e191-e203. doi:10.1016/S2214-109X(19)30482-6

356 2. Cohen PA, Jhingran A, Oaknin A, Denny L. Cervical cancer. The Lancet.

357 2019;393(10167):169-182. doi:10.1016/S0140-6736(18)32470-X

358 3. Monk BJ, Tewari KS, Koh W-J. Multimodality Therapy for Locally Advanced

359 Cervical Carcinoma: State of the Art and Future Directions. JCO. 2007;25(20):2952-

360 2965. doi:10.1200/JCO.2007.10.8324

361 4. Helmink BA, Khan MAW, Hermann A, Gopalakrishnan V, Wargo JA. The

362 microbiome, cancer, and cancer therapy. Nat Med. 2019;25(3):377-388.

363 doi:10.1038/s41591-019-0377-7

$3645 . \quad$ The Human Microbiome Project Consortium. Structure, function and diversity of

365 the healthy human microbiome. Nature. 2012;486(7402):207-214.

366 doi:10.1038/nature11234

367 6. Gopalakrishnan V, Spencer CN, Nezi L, et al. Gut microbiome modulates

368 response to anti-PD-1 immunotherapy in melanoma patients. Science.

369 2018;359(6371):97-103. doi:10.1126/science.aan4236

370 7. Chaput N, Lepage P, Coutzac C, et al. Baseline gut microbiota predicts clinical

371 response and colitis in metastatic melanoma patients treated with ipilimumab. Ann

372 Oncol. 2017;28(6):1368-1379. doi:10.1093/annonc/mdx108

373 8. Frankel AE, Coughlin LA, Kim J, et al. Metagenomic Shotgun Sequencing and

374 Unbiased Metabolomic Profiling Identify Specific Human Gut Microbiota and Metabolites 
375

376

377

378

379

380

381

382

383

384

385

386

387

388

389

390

391

392

393

394

395

396 Common Factor in Human Diseases. Biomed Res Int. 2017;2017.

397 doi:10.1155/2017/9351507

Associated with Immune Checkpoint Therapy Efficacy in Melanoma Patients.

Neoplasia. 2017;19(10):848-855. doi:10.1016/j.neo.2017.08.004

9. Vétizou M, Pitt JM, Daillère R, et al. Anticancer immunotherapy by CTLA-4 blockade relies on the gut microbiota. :7.

10. Sivan A, Corrales L, Hubert N, et al. Commensal Bifidobacterium promotes antitumor immunity and facilitates anti-PD-L1 efficacy. Science. 2015;350(6264):1084-

1089. doi:10.1126/science.aac4255

11. Chase D, Goulder A, Zenhausern F, Monk B, Herbst-Kralovetz M. The vaginal and gastrointestinal microbiomes in gynecologic cancers: A review of applications in etiology, symptoms and treatment. Gynecologic Oncology. 2015;138(1):190-200. doi:10.1016/j.ygyno.2015.04.036

12. Mitra A, Grossman Biegert GW, Delgado AY, et al. Microbial Diversity and Composition Is Associated with Patient-Reported Toxicity during Chemoradiation Therapy for Cervical Cancer. International Journal of Radiation

Oncology*Biology*Physics. January 2020. doi:10.1016/j.ijrobp.2019.12.040

13. Methé BA, Nelson KE, Pop $M$, et al. A framework for human microbiome research. Nature. 2012;486(7402):215-221. doi:10.1038/nature11209

14. Segata N, Izard J, Waldron L, et al. Metagenomic biomarker discovery and explanation. Genome Biology. 2011;12(6):R60. doi:10.1186/gb-2011-12-6-r60

15. Bunn A, Korpela M. An Introduction to dpIR. :16.

16. Rizzatti G, Lopetuso LR, Gibiino G, Binda C, Gasbarrini A. Proteobacteria: A 
398 17. Manichanh C, Varela E, Martinez C, et al. The Gut Microbiota Predispose to the

399 Pathophysiology of Acute Postradiotherapy Diarrhea. The American Journal of

400 Gastroenterology. 2008;103(7):1754-1761. doi:10.1111/j.1572-0241.2008.01868.x

401 18. Nam Y-D, Kim HJ, Seo J-G, Kang SW, Bae J-W. Impact of Pelvic Radiotherapy

402 on Gut Microbiota of Gynecological Cancer Patients Revealed by Massive

403 Pyrosequencing. Heimesaat MM, ed. PLoS ONE. 2013;8(12):e82659.

404 doi:10.1371/journal.pone.0082659

405 19. Wang A, Ling Z, Yang Z, et al. Gut Microbial Dysbiosis May Predict Diarrhea and 406 Fatigue in Patients Undergoing Pelvic Cancer Radiotherapy: A Pilot Study. Grivennikov

407 S, ed. PLoS ONE. 2015;10(5):e0126312. doi:10.1371/journal.pone.0126312

408 20. Sethi V, Kurtom S, Tarique M, et al. Gut Microbiota Promotes Tumor Growth in

409 Mice by Modulating Immune Response. Gastroenterology. 2018;155(1):33-37.e6.

410 doi:10.1053/j.gastro.2018.04.001

411 21. Weingarden A, González A, Vázquez-Baeza Y, et al. Dynamic changes in short-

412 and long-term bacterial composition following fecal microbiota transplantation for

413 recurrent Clostridium difficile infection. Microbiome. 2015;3(1):10. doi:10.1186/s40168-

$414 \quad 015-0070-0$

415 22. Ahn J, Sinha R, Pei Z, et al. Human Gut Microbiome and Risk for Colorectal

416 Cancer. JNCl: Journal of the National Cancer Institute. 2013;105(24):1907-1911.

417 doi:10.1093/jnci/djt300

418 23. Baxter NT, Zackular JP, Chen GY, Schloss PD. Structure of the gut microbiome

419 following colonization with human feces determines colonic tumor burden. Microbiome.

$420 \quad 2014 ; 2(1): 20$. doi:10.1186/2049-2618-2-20 
421 24. Song Y, Garg S, Girotra M, et al. Microbiota dynamics in patients treated with

422 fecal microbiota transplantation for recurrent Clostridium difficile infection. PLoS ONE.

423 2013;8(11):e81330. doi:10.1371/journal.pone.0081330

424 25. Yeung AR, Pugh SL, Klopp AH, et al. Improvement in Patient-Reported

425 Outcomes With Intensity-Modulated Radiotherapy (RT) Compared With Standard RT: A

426 Report From the NRG Oncology RTOG 1203 Study. JCO. February

427 2020:JCO.19.02381. doi:10.1200/JCO.19.02381

428 26. Wang $\mathrm{Y}$, Wiesnoski DH, Helmink BA, et al. Fecal microbiota transplantation for

429 refractory immune checkpoint inhibitor-associated colitis. Nat Med. 2018;24(12):1804-

430 1808. doi:10.1038/s41591-018-0238-9

431 27. Chen D, Wu J, Jin D, Wang B, Cao H. Fecal microbiota transplantation in cancer

432 management: Current status and perspectives. International Journal of Cancer.

433 2019;145(8):2021-2031. doi:10.1002/ijc.32003 
El Alam et al 2020

435 Table 1. Differences between baseline diversity metrics and those assessed during and

436 after chemoradiotherapy

Diversity metric Time point $\quad$ Mean \pm SD $\quad P^{a}$

\begin{tabular}{|c|c|c|c|}
\hline \multirow[t]{5}{*}{ Observed OTUs } & Baseline & $107.58 \pm 34.60$ & - \\
\hline & Week 1 & $101.74 \pm 32.38$ & 0.625 \\
\hline & Week 3 & $99.08 \pm 25.02$ & 0.064 \\
\hline & Week 5 & $83.79 \pm 26.28$ & $<0.001$ \\
\hline & Week 12 & $104.00 \pm 32.98$ & 0.285 \\
\hline \multirow[t]{5}{*}{ Shannon } & Baseline & $2.91 \pm 0.59$ & - \\
\hline & Week 1 & $2.92 \pm 0.46$ & 0.599 \\
\hline & Week 3 & $2.93 \pm 0.45$ & 0.400 \\
\hline & Week 5 & $2.52 \pm 0.68$ & $<0.001$ \\
\hline & Week 12 & $3.01 \pm 0.48$ & 0.614 \\
\hline \multirow[t]{5}{*}{ Simpson } & Baseline & $0.87 \pm 0.09$ & - \\
\hline & Week 1 & $0.89 \pm 0.06$ & 0.566 \\
\hline & Week 3 & $0.89 \pm 0.06$ & 0.844 \\
\hline & Week 5 & $0.81 \pm 0.15$ & 0.002 \\
\hline & Week 12 & $0.89 \pm 0.07$ & 0.368 \\
\hline
\end{tabular}




\begin{tabular}{|c|c|c|c|}
\hline \multirow[t]{5}{*}{ Inverse Simpson } & Baseline & $11.39 \pm 6.55$ & \\
\hline & Week 1 & $10.63 \pm 4.17$ & 0.326 \\
\hline & Week 3 & $11.75 \pm 5.95$ & 0.441 \\
\hline & Week 5 & $8.23 \pm 5.05$ & 0.001 \\
\hline & Week 12 & $11.54 \pm 4.42$ & 0.277 \\
\hline \multirow[t]{5}{*}{ Fisher } & Baseline & $18.26 \pm 6.98$ & - \\
\hline & Week 1 & $17.07 \pm 6.5$ & 0.637 \\
\hline & Week 3 & $16.45 \pm 5.00$ & 0.053 \\
\hline & Week 5 & $13.53 \pm 4.95$ & $<0.001$ \\
\hline & Week 12 & $17.52 \pm 6.51$ & 0.266 \\
\hline
\end{tabular}


439 Table 2. Results of the linear mixed model analysis of time as a predictor of diversity,

440 accounting for patients with missing data for one or more time points ${ }^{a}$

\section{Outcome Covariate $\quad$ Estimate $95 \%$ confidence interval $P$}

\begin{tabular}{|c|c|c|c|c|}
\hline \multirow[t]{3}{*}{ Observed OTUs } & Low Diversity & -51.106 & $(-61.79,-40.42)$ & $<0.01$ \\
\hline & Time & -7.569 & $(-9.66,-5.47)$ & $<0.01$ \\
\hline & Low Diversity:Time & 6.403 & $(3.36,9.48)$ & $<0.01$ \\
\hline \multirow[t]{3}{*}{ Shannon } & Low Diversity & -0.80802 & $(-1.03,-0.59)$ & $<0.01$ \\
\hline & Time & -0.1181 & $(-0.16,-0.07)$ & $<0.01$ \\
\hline & Low Diversity:Time & 0.08781 & $(0.02,0.15)$ & $<0.01$ \\
\hline \multirow[t]{3}{*}{ Simpson } & Low Diversity & -0.091656 & $(-0.13,-0.05)$ & $<0.01$ \\
\hline & Time & -0.013607 & $(-0.02,0)$ & $<0.01$ \\
\hline & Low Diversity:Time & 0.002634 & $(-0.01,0.02)$ & 0.684 \\
\hline \multirow[t]{3}{*}{ Inverse Simpson } & Low Diversity & -8.1326 & $(-10.32,-5.95)$ & $<0.01$ \\
\hline & Time & -1.1006 & $(-1.56,-0.64)$ & $<0.01$ \\
\hline & Low Diversity:Time & 1.1159 & $(0.45,1.78)$ & $<0.01$ \\
\hline \multirow[t]{3}{*}{ Fisher } & Low Diversity & -10.5757 & $(-12.66,-8.49)$ & $<0.01$ \\
\hline & Time & -1.6527 & $(-2.08,-1.22)$ & $<0.01$ \\
\hline & Low Diversity:Time & 1.453 & $(0.86,2.05)$ & $<0.01$ \\
\hline
\end{tabular}


bioRxiv preprint doi: https://doi.org/10.1101/2020.04.10.036426; this version posted April 12, 2020. The copyright holder for this preprint (which was not certified by peer review) is the author/funder. All rights reserved. No reuse allowed without permission.

El Alam et al 2020

25

441 aSamples collected 12 weeks after treatment initiation were excluded from the analysis. 
443 Supplemental Table 1. Patient demographic and clinical characteristics ( $N=58$ )

\begin{tabular}{lll} 
& $\mathrm{N}$ & $(\%)$ \\
\hline Mean age (SD) & $49.36(10.52)$ & - \\
\hline BMI, Mean (SD), kg/m2 & $28.7(6.07)$ & - \\
\hline Type of Cancer & & \\
\hline Cervical & 55 & $(94.8)$ \\
\hline Vaginal & 2 & $(3.4)$ \\
\hline Vulvar & 1 & $(1.7)$
\end{tabular}

\section{Race/Ethnicity}

$\begin{array}{cll}\text { Asian } & 2 & (3.5) \\ \text { Black } & 4 & (7.0) \\ \text { Hispanic } & 26 & (45.6) \\ \text { White } & 24 & (42.1) \\ \text { Other } & 1 & (1.8)\end{array}$

FIGO Stage

\begin{tabular}{llll}
\hline IA1 & 1 & $(1.7)$ \\
IA2 & 0 & $(0)$ \\
IB1 & 6 & $(10.3)$ \\
IB2 & 6 & $(10.3)$ \\
IIA & 3 & $(5.2)$ \\
IIB & 28 & $(48.3)$ \\
IIIA & 9 & $(15.5)$ \\
IIIB & 0 & $(0)$ \\
IVA & 4 & $(6.9)$ \\
IVB & 1 & $(1.7)$ \\
\hline Histology & Well & 3 & $(5.2)$ \\
\hline Moderate & 20 & $(34.5)$ \\
Poor & 26 & $(44.8)$ \\
Adenocarcinoma & 10 & $(15.5)$ \\
Adenosquamous & 2 & $(3.4)$ \\
\hline
\end{tabular}


Node Level on PET

\begin{tabular}{rll}
\hline Common lliac & 9 & $(15.5)$ \\
External Iliac & 23 & $(39.7)$ \\
Internal Iliac & 6 & $(10.3)$ \\
Para-Aortic & 3 & $(5.2)$ \\
None & 17 & $(29.3)$ \\
Smoking status & & \\
\hline Current & 5 & $(8.8)$ \\
Former & 21 & $(36.8)$ \\
Never & 31 & $(54.4)$ \\
Antibiotic Use & & \\
\hline No & 16 & $(27.6)$ \\
Yes & 42 & $(72.4)$ \\
Brachytherapy & & \\
\hline HDR & 21 & $(38.2)$ \\
PDR & 34 & $(61.8)$ \\
HPV Status & & $(77.6)$ \\
\hline Positive & 45 & $(17.2)$ \\
Negative & $10.2)$
\end{tabular}


445 Supplemental Table 2. Samples available for each patient at each time point.

\begin{tabular}{|c|c|c|c|c|c|}
\hline Patient \# & Baseline & Week 1 & Week 3 & Week 5 & Week 12 \\
\hline 1 & & & & & \\
\hline 2 & & & & & \\
\hline 3 & & & & & \\
\hline 4 & & & & & \\
\hline 5 & & & & & \\
\hline 6 & & & & & \\
\hline 7 & & & & & \\
\hline 8 & & & & & \\
\hline 9 & & & & & \\
\hline 10 & & & & & \\
\hline 11 & & & & & \\
\hline 12 & & & & & \\
\hline 13 & & & & & \\
\hline 14 & & & & & \\
\hline 15 & & & & & \\
\hline 16 & & & & & \\
\hline 17 & & & & & \\
\hline 18 & & & & & \\
\hline 19 & & & & & \\
\hline 20 & & & & & \\
\hline 21 & & & & & \\
\hline 22 & & & & & \\
\hline 23 & & & & & \\
\hline 24 & & & & & \\
\hline 25 & & & & & \\
\hline 26 & & & & & \\
\hline 27 & & & & & \\
\hline 28 & & & & & \\
\hline
\end{tabular}


bioRxiv preprint doi: https://doi.org/10.1101/2020.04.10.036426; this version posted April 12, 2020. The copyright holder for this preprint (which was not certified by peer review) is the author/funder. All rights reserved. No reuse allowed without permission.

El Alam et al 2020

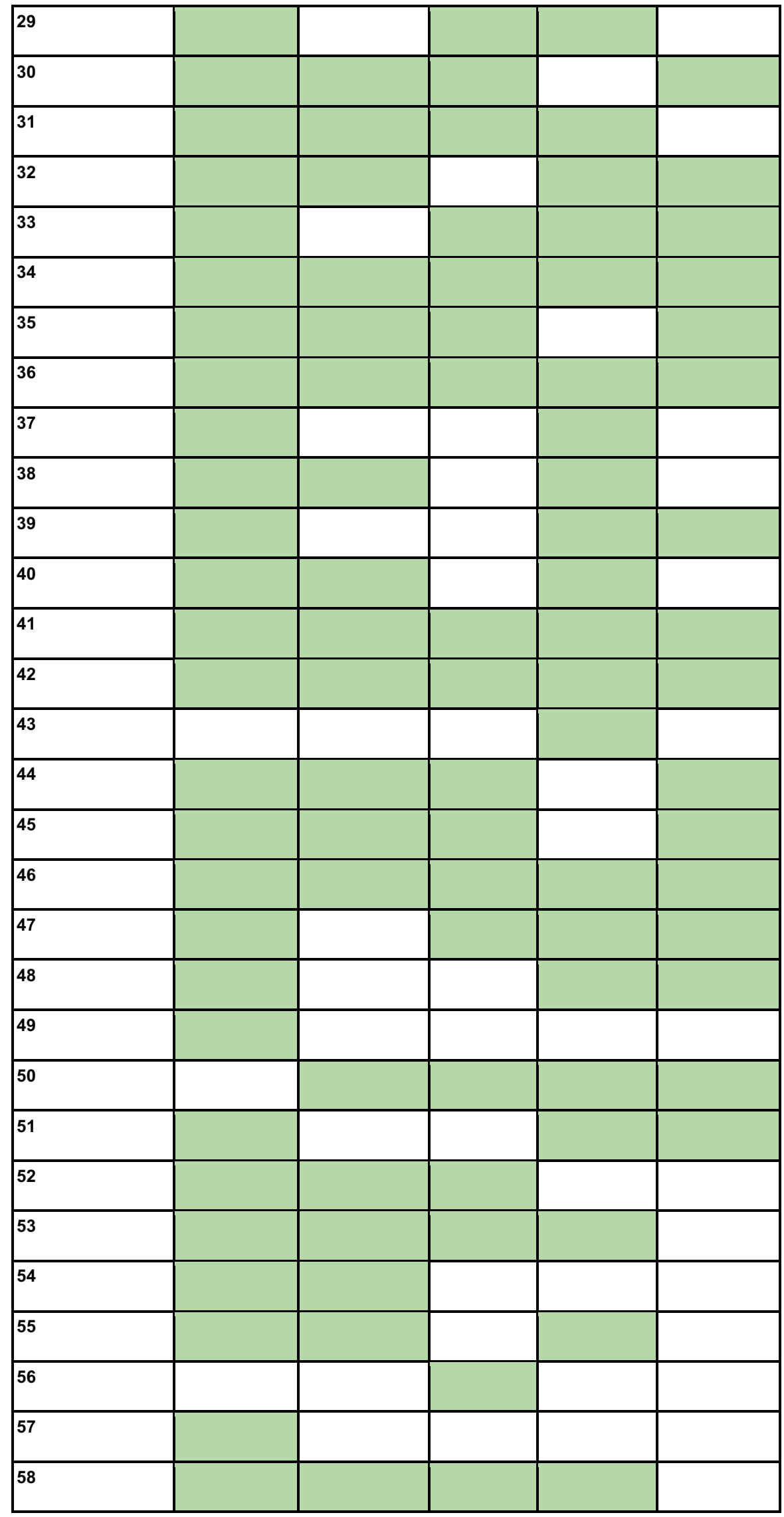


bioRxiv preprint doi: https://doi.org/10.1101/2020.04.10.036426; this version posted April 12, 2020. The copyright holder for this preprint (which was not certified by peer review) is the author/funder. All rights reserved. No reuse allowed without permission.

El Alam et al 2020

446 


\section{Figure Legends}

448 Figure 1. Gut richness and diversity decrease significantly by week 5 of

449 chemoradiotherapy. Box plots show changes in (A) observed OTUs (richness) and in

450 (B) Shannon, (C) Fisher, (D) Simpson, and (E) Inverse Simpson diversity indices over

451 time as determined with repeated measures ANOVA. P-values $>0.05$ are not shown.

452 Figure 2. Gut richness and diversity are at or near baseline levels by first follow-

453 up after chemoradiotherapy. Individual richness and diversity values are plotted for all

454 patients who provided samples at baseline, week 5, and week 12. Each line denotes an

455 individual patient. Most patients' gut diversity and richness returned or nearly returned

456 to baseline levels. Green represents patients whose week 12 diversity levels were equal

457 to or greater than baseline diversity levels. Black represents patients whose diversity

458 levels decreased by week 5 but increased to near-baseline levels by week 12. Red

459 represents patients whose diversity levels declined continuously from baseline to 12

460 weeks.

461 Figure 3. Patients with high baseline gut richness and diversity have significantly

462 greater decreases in diversity over time. Linear mixed modeling demonstrated an

463 interaction between time and baseline diversity values for all metrics except Simpson

464 diversity. Patients with high baseline diversity (red) had a significantly steeper decline in

465 diversity than patients with low baseline diversity (blue) did.

466 Figure 4. Gut composition changes significantly during chemoradiotherapy,

467 adapts after treatment, and remains altered at first follow-up. The differential

468 enrichments of bacterial phyla, classes, orders, families, and genera between baseline

469 and week 5 were identified by linear discriminant analysis effect size. Significant

470 differences are those with a logarithmic linear discriminant analysis score $>4$ and a 
471 factorial Kruskal-Wallis $p$-value $<0.05$. Bacteria that were most significantly altered

472 during chemoradiotherapy (between baseline and week 5; A and B) included the

473 Clostridia class and Proteobacteria phylum. After chemoradiotherapy (between week 5

474 and week 12; C), proteobacteria decreased, whereas Ezakiella increased. Overall, the

475 most significant alteration between baseline and first chemoradiotherapy follow-up

476 (week 12; D) was an increase in Bacteroidales.

477 Figure 5. Significant adaptations in gut composition occur during and after 478 chemoradiotherapy. The relative abundances of the pathogenic, gram-negative

479 Proteobacteria phylum (A) increased significantly, whereas those of the beneficial 480 members of the Clostridiales class (B) decreased significantly, during

481 chemoradiotherapy but tended to return or nearly return to baseline levels by first follow482 up (week 12). The most significant alteration between baseline and week 12 was an 483 increase in the generally beneficial Bacteroidetes $(C)$, which are known to help prevent 484 pathogenic bacteria from colonizing the human gut and may be a sign of adaptation in 485 response to increases in pathogenic bacteria during chemoradiotherapy. Enrichment 486 analysis of rare species (D) suggests adaptation of individual microbial species at the 487 OTU level. For example, individual species of Clostridia $(E)$ were either enriched or 488 depleted at week 5 as compared with baseline.

489 Supplementary Figure 1. Cumulative patients receiving antibiotics. Number of 490 patients who took antibiotics prior to each time point are presented. Total number of 491 patients receiving antibiotics during the study period is 42 .

492 Supplementary Figure 2. Changes in relative abundances of different species 493 between baseline and week 5 . Relative abundances of species identified using linear 
494 discriminant analysis effect size as having changed between baseline and week 5.

495 Whiskers on the plot represent the confidence interval.

\section{Supplementary Figure 3. Changes in relative abundances of species between}

497 baseline and week 12. Relative abundances of species identified using linear

498 discriminant analysis effect size as having changed between baseline and week 5 are

499 plotted. Whiskers on the plot represent the confidence interval. 
A bioRxiv preprint doi: https://doi.org/10.1101/2020.04.10.036426; this vession posted April 12, 2020. The copyright holder for this preprint (which

A was not certified by peer review) is the author/funder. AB rights reserved. No reuse allowed without permission.
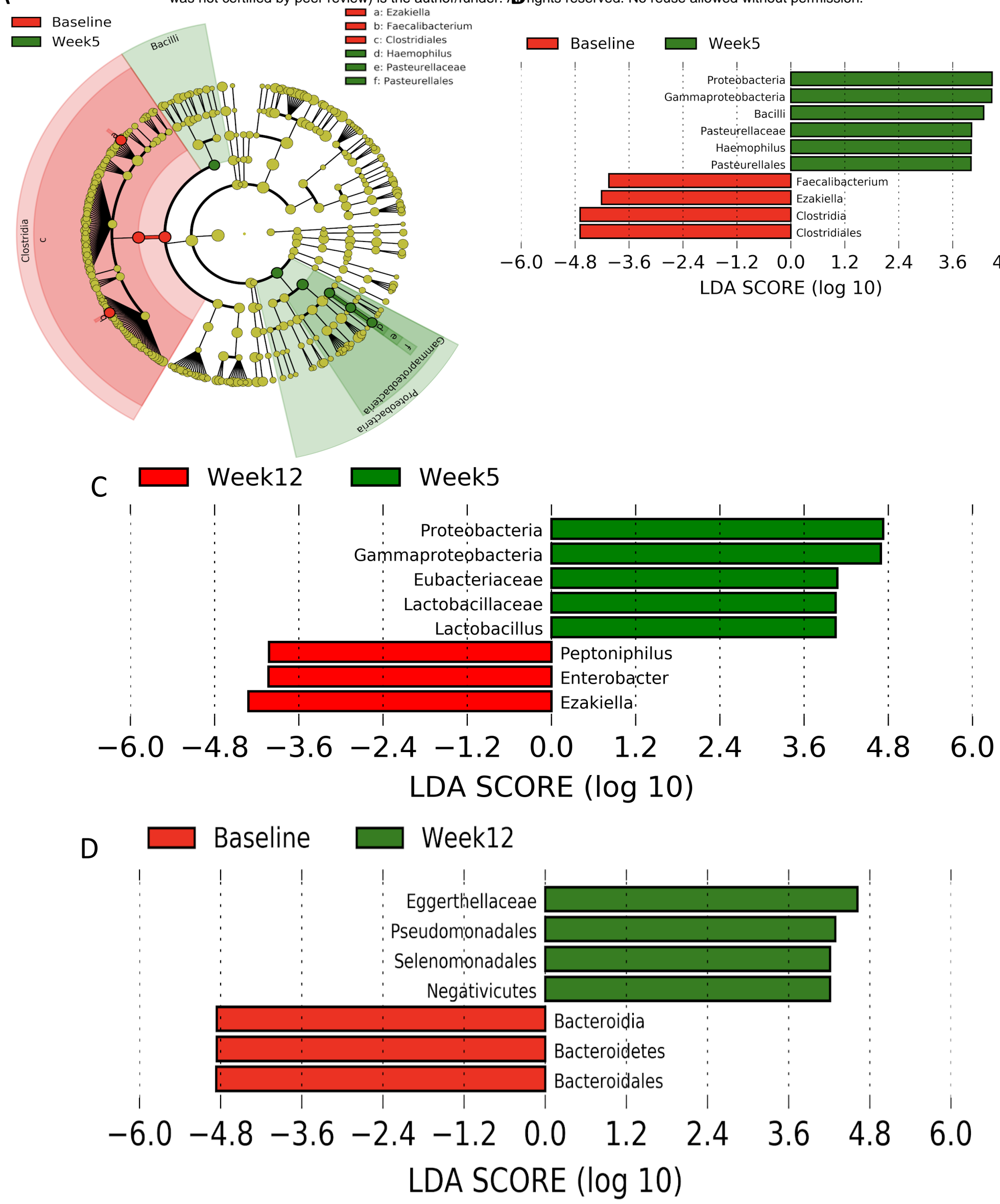

Figure 4. Gut composition changes significantly during chemoradiotherapy, adapts after treatment, and remains altered at first follow-up. The differential enrichments of bacterial phyla, classes, orders, families, and genera between baseline and week 5 were identified by linear discriminant analysis effect size. Significant differences are those with a logarithmic linear discriminant analysis score $>4$ and a factorial Kruskal-Wallis $p$-value $<0.05$. Bacteria that were most significantly altered during chemoradiotherapy (between baseline and week 5; A and B) included the Clostridia class and Proteobacteria phylum. After chemoradiotherapy (between week 5 and week 12; C), proteobacteria decreased, whereas Ezakiella increased. Overall, the most significant alteration between baseline and first chemoradiotherapy follow-up (week 12; D) was an increase in Bacteroidales. 


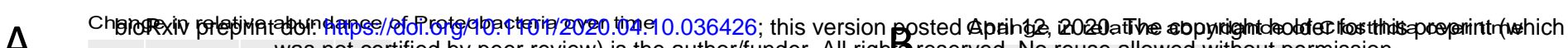
was not certified by peer review) is the author/funder. All righ $\mathbf{B}$ reserved. No reuse allowed without permission.

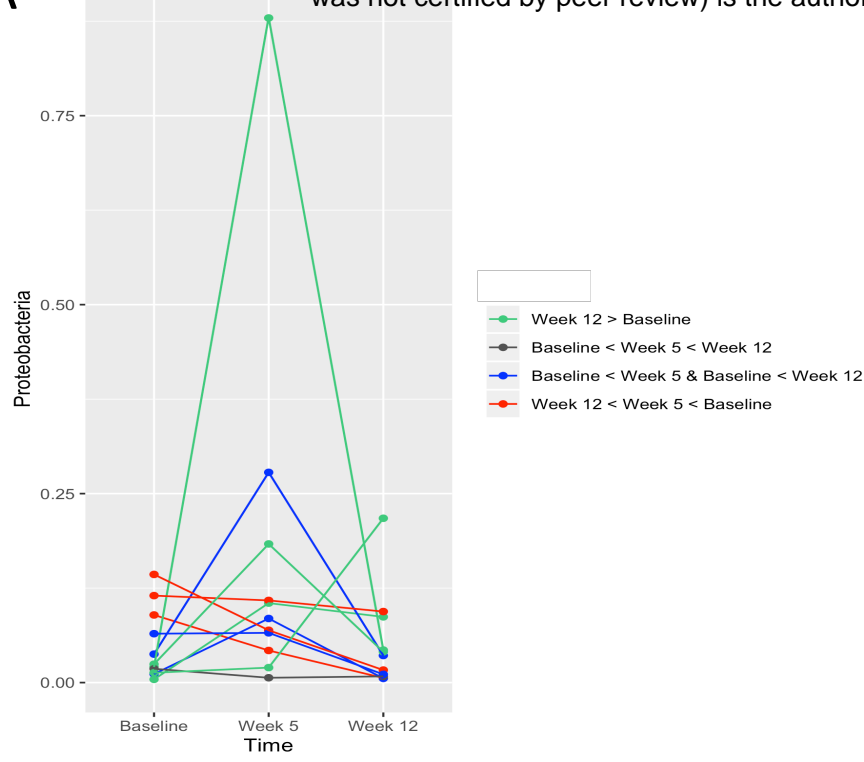

C

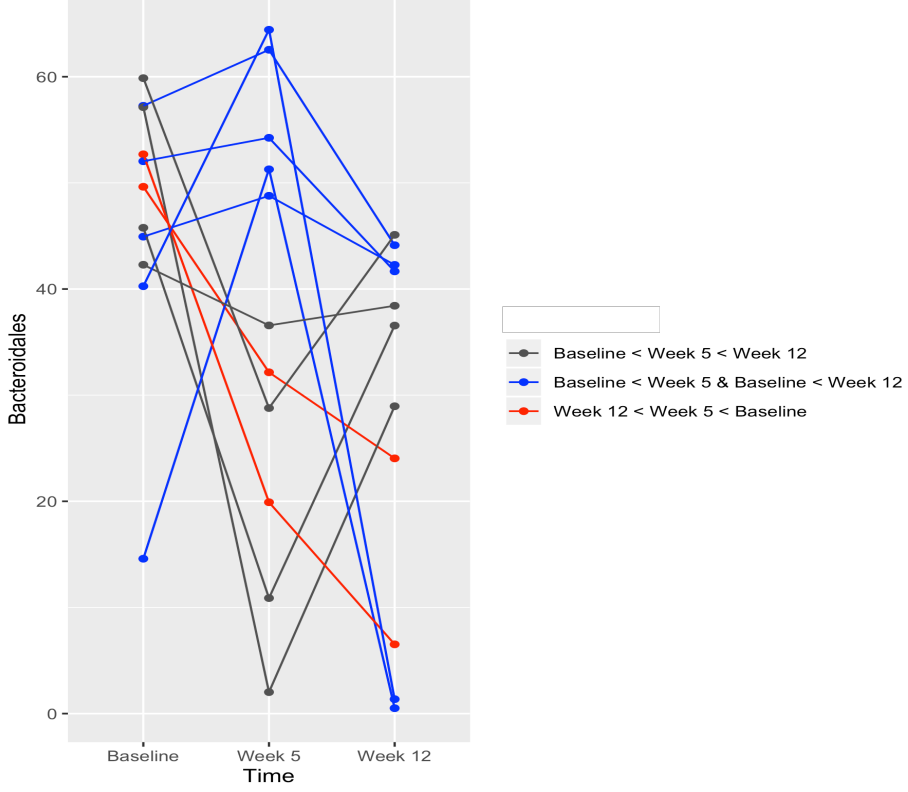

E

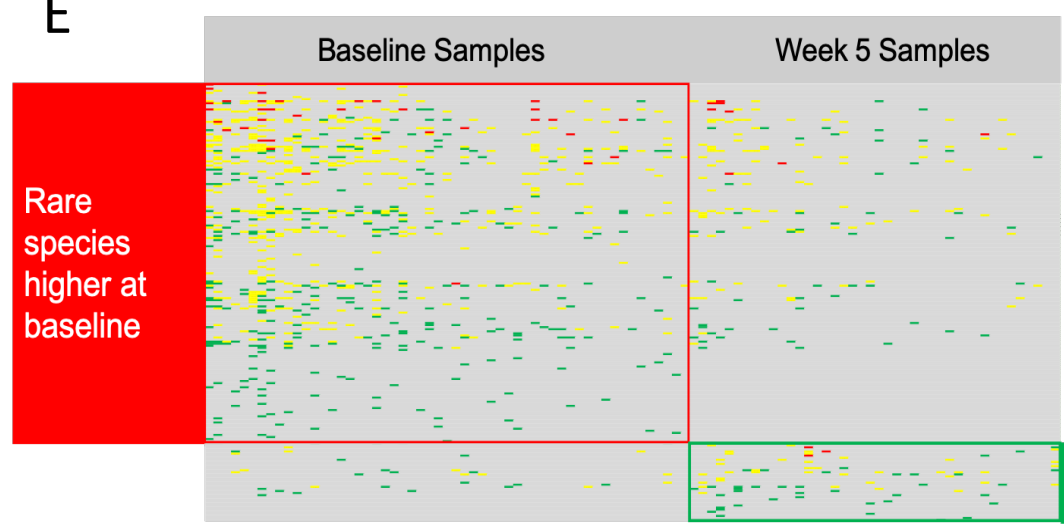

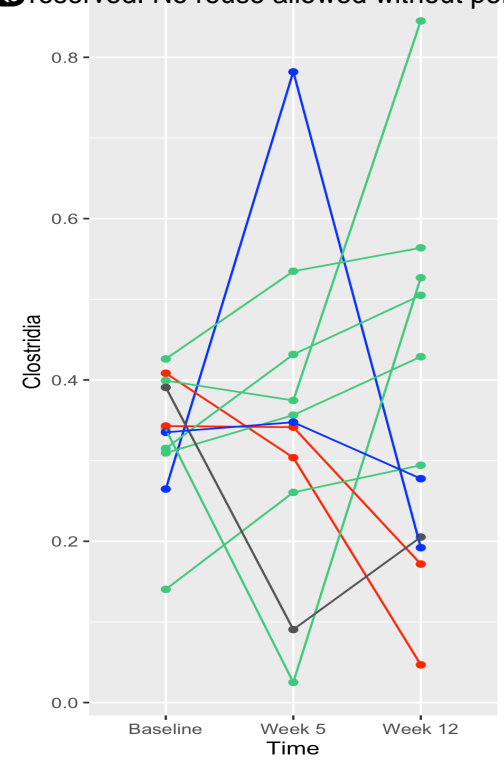

- Week $12>$ Baseline

- Baseline $<$ Week $5<$ Week 12

$\rightarrow$ Baseline $<$ Week 5 \& Baseline < Week 12

$\rightarrow$ Week $12<$ Week $5<$ Baseline
D

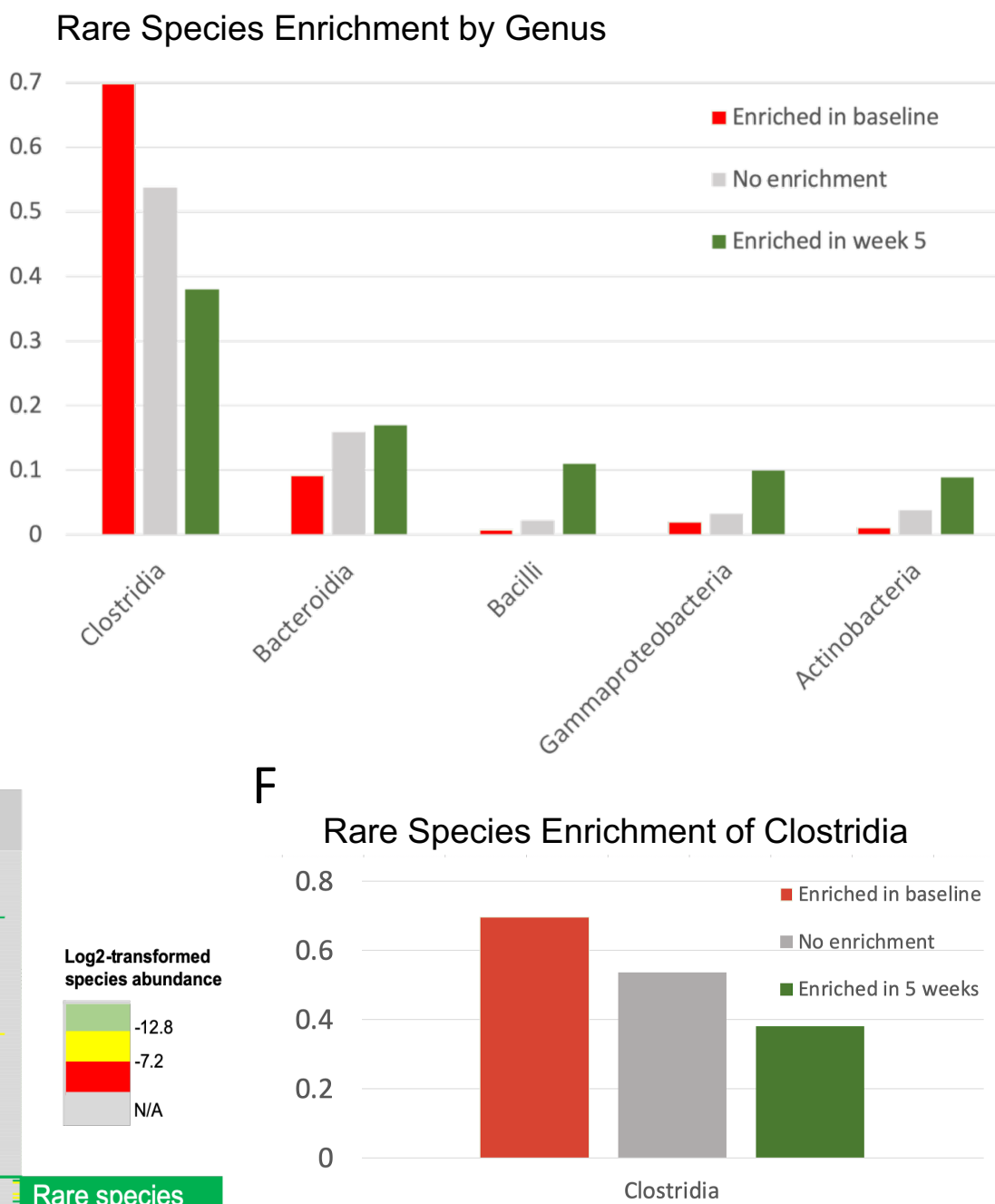

Figure 5. Significant adaptations in gut composition occur during and after chemoradiotherapy. The relative abundances of the pathogenic, gram-negative Proteobacteria phylum $(A)$ increased significantly, whereas those of the beneficial members of the Clostridiales class (B) decreased significantly, during chemoradiotherapy but tended to return or nearly return to baseline levels by first follow-up (week 12). The most significant alteration between baseline and week 12 was an increase in the generally beneficial Bacteroidetes (C), which are known to help prevent pathogenic bacteria from colonizing the human gut and may be a sign of adaptation in response to increases in pathogenic bacteria during chemoradiotherapy. Enrichment analysis of rare species (D) suggests adaptation of individual microbial species at the OTU level. For example, individual species of Clostridia (E) were either enriched or depleted at week 5 as compared with baseline. 
Cumulative patients receiving antibiotics

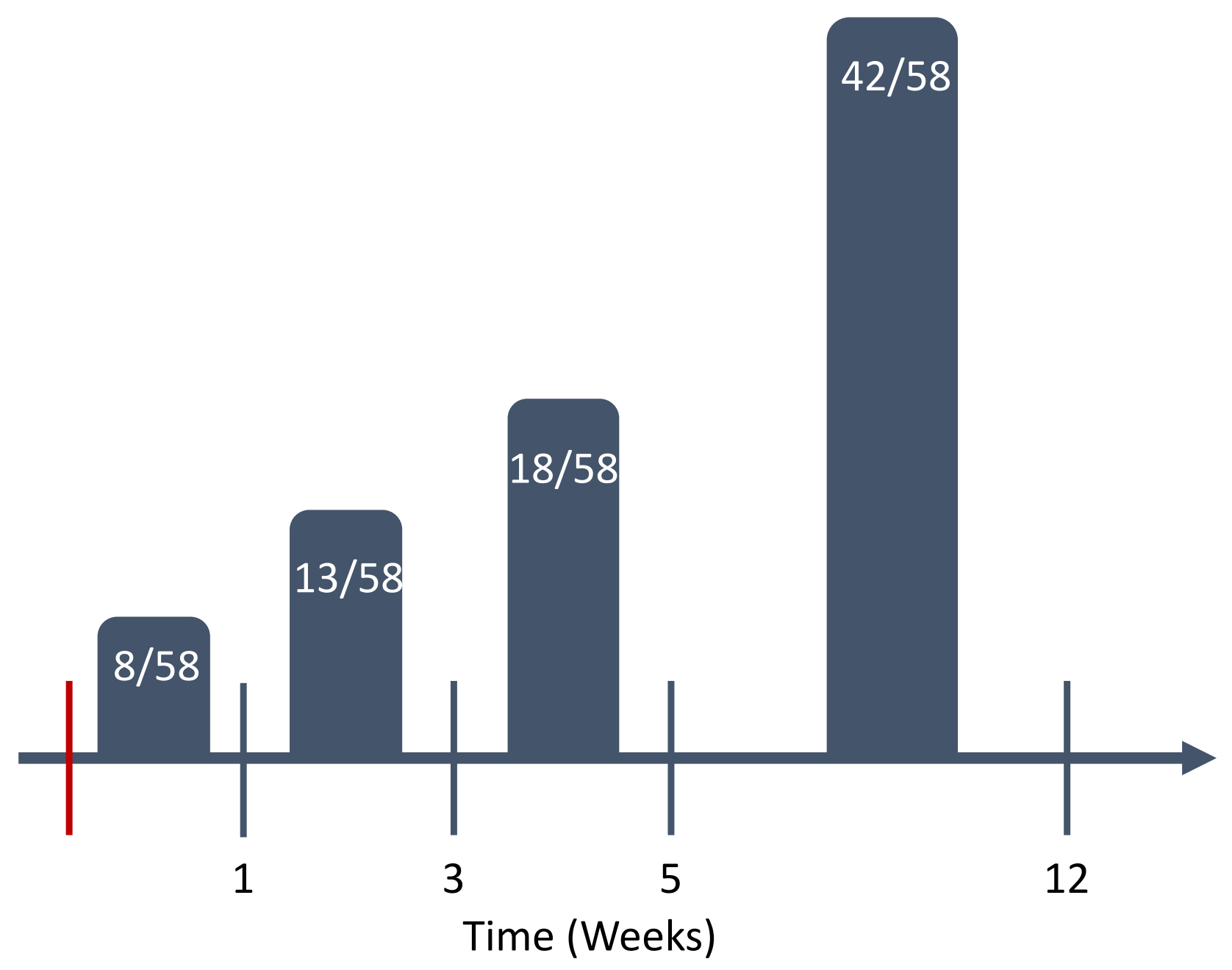

Supplementary Figure 1. Cumulative patients receiving antibiotics. Number of patients who took antibiotics prior to each time point are presented. Total number of patients receiving antibiotics during the study period is 42 . 
